\title{
Impacto metabólico e inflamatorio de una comida rica en grasas saturadas y su relación con la obesidad abdominal
}

\author{
Alicia Norma Alayón ${ }^{1,2}$, Ana Patricia Rivadeneira², Carlos Herrera², Heidy Guzmán², \\ Dioneris Arellano², Isabella Echeverri ${ }^{3}$ \\ Grupo de Nutrición, Doctorado en Ciencias Biomédicas, Universidad del Valle, Cali, Colombia \\ 2 Grupo de Investigaciones Biomédicas, Universidad de San Buenaventura, Cartagena, Colombia \\ 3 Grupo de Investigación Biomédica, Facultad de Ciencias de la Salud, Universidad ICESI, Cali, Colombia
}

Introducción. La etapa posprandial se asocia con el incremento de marcadores relacionados con el riesgo cardiovascular, cuya intensidad depende del estado metabólico.

Objetivo. Determinar el impacto de la ingestión de una comida rica en grasas saturadas sobre el perfil metabólico e inflamatorio y su relación con la obesidad abdominal.

Materiales y métodos. Se hizo un ensayo clínico en 42 individuos (21 con obesidad abdominal). Se midieron, en sangre, la glucosa, la insulina, el perfil lipídico, la proteína $\mathrm{C}$ reactiva, los lipopolisacáridos y la interleucina 6 , en ayunas y después de la ingestión.

Resultados. Además de la obesidad, se registró la presencia de resistencia a la insulina y de niveles elevados de triacilglicéridos y proteína $C$ reactiva en ayunas. Asimismo, se detectaron niveles posprandiales más elevados de glucosa, insulina y triacilglicéridos. La interleucina 6 disminuyó en el grupo de personas sin obesidad y los lipopolisacáridos aumentaron en ambos grupos.

Conclusión. La ingestión de una comida rica en grasas saturadas produjo un mayor impacto en las variables glucémicas en el grupo con obesidad y, aunque afectó de forma similar los lípidos en ambos grupos, el incremento de triacilglicéridos fue mayor en presencia de una concentración basal elevada y promovió el aumento de lipopolisacáridos. El estado inflamatorio basal y posprandial afectó en mayor medida al grupo con obesidad. El momento posprandial reflejó el estado más frecuente de los individuos en un día normal y permitió evidenciar la capacidad de respuesta metabólica frente a la ingestión de alimentos, así como los estados tempranos de riesgo metabólico.

Palabras clave: obesidad; inflamación; periodo posprandial; dieta rica en grasa; resistencia a la insulina; lipopolisacáridos.

doi: https://doi.org/10.7705/biomedica.v38i0.3911

\section{Metabolic and inflammatory postprandial effect of a highly saturated fat meal and its relationship to abdominal obesity}

Introduction: The postprandial stage is associated with the increase of markers related to cardiovascular risk, and its intensity depends on the metabolic state.

Objective: To determine the impact of a high-fat meal intake on the metabolic and inflammatory profile, and its relationship to abdominal obesity.

Materials and methods: This clinical trial included 42 individuals ( 21 with abdominal obesity). We measured glucose, insulin, lipid profile, reactive C protein, lipopolysaccharides, and interleukin 6 in fasting blood, and four hours after eating.

Results: Besides obesity, we found insulin resistance and higher levels of fasting triacylglycerides and C-reactive protein. There were higher postprandial responses to glucose, insulin, and triacylglycerides. Interleukin 6 decreased in the non-obese group, and lipopolysaccharides increased in both groups.

Conclusions: A saturated high-fat food intake produced a greater impact on the glycemic variables in the group with obesity, while it affected the lipids in both groups. However, the increase of triacylglycerides was higher in the presence of a high basal concentration, and it promoted the increase of lipopolysaccharides. The basal and postprandial inflammatory state affected the group with obesity more. The postprandial moment reflected the most frequent state of the individuals on a normal day and evidenced the capacity of the metabolic response to food intake, as well as early metabolic risk states.

Key words: Obesity; inflammation; postprandial period; diet, high-fat; insulin resistance; lipopolysaccharides.

doi: https://doi.org/10.7705/biomedica.v38i0.3911

\section{Contribución de los autores:}

Todos los autores contribuyeron a la recolección, análisis e interpretación de los datos y a la escritura del manuscrito. 
La ingestión de alimentos y el procesamiento y aprovechamiento de los nutrientes son elementos clave para la supervivencia, pero también constituyen un reto para la homeostasis metabólica (1). En el momento posprandial aparecen estados proinflamatorios, cuya magnitud varía según la presencia de comorbilidades, como la obesidad abdominal y el tipo de alimento ingerido $(2,3)$. Dicho estado se caracteriza por el aumento en la circulación de lipoproteínas ricas en triacilglicéridos y lipopolisacáridos, los cuales se relacionan con el aumento de marcadores inflamatorios (4) y la formación de células espumosas (5), y contribuyen al desarrollo de enfermedades cardiovasculares.

En los estudios en ciudades de Colombia, entre ellas Cartagena, se ha evidenciado la transición demográfica y nutricional por la que el país transita, caracterizada por una tendencia creciente a la prevalencia de obesidad (6) y de enfermedades cardiovasculares que han pasado a ocupar los primeros lugares en morbilidad y mortalidad $(7,8)$. Sumado a ello, los hábitos de nuestra población llevan a que un individuo promedio pase la mayor parte del día en estado posprandial, y tenga consumos hipercalóricos y poca actividad física (9). Dichas circunstancias motivaron esta investigación, cuyo objetivo fue conocer de qué manera la ingestión de una comida estandarizada, rica en grasas saturadas, modifica los perfiles metabólicos de glúcidos y lípidos, y promueve estados de inflamación, así como la influencia de la obesidad abdominal en ese contexto.

\section{Materiales y métodos}

Se llevó a cabo un ensayo clínico en 42 participantes adultos, escogidos por muestreo aleatorio entre los empleados de una universidad de la ciudad, a quienes previamente se les había hecho una entrevista para verificar los criterios de inclusión (sexo masculino, bajo nivel de actividad física y aparentemente sanos). Se excluyeron del estudio a los fumadores, a las personas en dieta o que tomaran medicamentos o vitaminas, a quienes tenían un consumo elevado de alcohol, o registraran concentraciones de proteína $\mathrm{C}$ reactiva mayores de $10 \mathrm{mg} / \mathrm{l}$.

\footnotetext{
$\overline{\text { Correspondencia: }}$

Alicia Norma Alayón, Grupo de Investigaciones Biomédicas, Universidad de San Buenaventura, Diagonal 32 №30-966, Cartagena, Colombia

Teléfono: (575) 653 5555, extensión 202

aalayon@usbctg.edu.co; alinorala@gmail.com

Recibido: 24/05/17; aceptado: 14/08/17
}

A los participantes se les solicitó que no hicieran cambios en sus estilos de vida durante la semana anterior al estudio y que observaran 12 horas de ayuno antes de la toma de la muestra de sangre basal, y dos más una hora y cuatro horas después de ingerir un desayuno que incluía $100 \mathrm{~g}$ de pan untado con $70 \mathrm{~g}$ de mantequilla y un café azucarado, el cual aportaba 874 calorías, $59 \%$ de las cuales provenían de grasas (saturadas, $32 \%$, monoinsaturadas, $23 \%$, y poliinsaturadas, $4 \%$ ), $37 \%$ de hidratos de carbono y $4 \%$ de proteínas. Durante la espera se les permitió beber agua, pero no consumir otros alimentos ni realizar ejercicio físico. El tiempo de seguimiento se estableció considerando los intervalos de cuatro horas en promedio, habituales entre comidas.

Los sueros se separaron y se conservaron a $-20^{\circ} \mathrm{C}$ para la posterior cuantificación de la glucosa, la insulina, el colesterol total y la fracción HDL (C-HDL), los triacilglicéridos, los lipopolisacáridos, la interleucina 6 (IL-6) y la proteína C reactiva.

Las concentraciones de glucosa y el perfil lipídico se midieron mediante métodos enzimáticos espectrofotométricos, utilizando estuches comerciales (Human). Para la medición de la insulina y la IL-6, se utilizaron técnicas de inmunoensayo enzimático: Insulin-AccuBind ${ }^{\mathrm{TM}}$ y Human IL-6 High SensitivityeBioscience $^{\mathrm{TM}}$, respectivamente. El índice HOMA-IR (Homeostatic Model Assessment) se calculó según la siguiente fórmula: insulina $(\mu \mathrm{Ul} / \mathrm{ml}) \times$ glucosa $(\mathrm{mmol} / \mathrm{l})$ / 22,5. La proteína $C$ reactiva se determinó mediante el método turbidimétrico cuantitativo de alta sensibilidad (Spinreact). Las muestras en ayunas y a las cuatro horas para la medición de los marcadores inflamatorios se tomaron en brazos distintos y en venas de fácil acceso, con el fin de controlar posibles contribuciones inespecíficas derivadas de la punción.

Todo el material para la determinación de los lipopolisacáridos se certificó como apirógeno, utilizando el método cromogénico de punto final (QCL-1000 Endpoint Chromogenic LAL Assays ${ }^{\mathrm{TM}}$ Lonza), previa dilución del suero en agua libre de pirógenos $(1 / 20)$ e inactivación a $75^{\circ} \mathrm{C}$ durante 15 minutos. En todas las pruebas, las curvas estándares tuvieron un coeficiente de correlación superior a 0,97. Para controlar las reacciones de inhibición, se adicionó una cantidad conocida de lipopolisacáridos $(0,4 \mathrm{EU} / \mathrm{ml})$, con lo cual se logró una recuperación de $108 \%$, acorde con el estándar de calidad establecido por el fabricante ( $\pm 25 \%)$. Las pruebas se llevaron a cabo en los laboratorios 
de atención a la comunidad y de investigaciones de la Universidad de San Buenaventura en Cartagena, y se ajustaron a los estándares y controles de calidad requeridos para asegurar la validez y la confiabilidad de los resultados según las especificaciones de los fabricantes.

\section{Análisis estadístico}

El número de participantes se calculó con la fórmula para la comparación de medidas repetidas en dos grupos y un $15 \%$ adicional para prever posibles pérdidas.

El impacto global de la intervención se midió como el área bajo la curva (Area under the Curve, AUC) calculada con el método trapezoidal, y el aumento sobre el nivel basal se estimó como el incremento del área bajo la curva (Incremental Area under the Curve, iAUC), sustrayendo previamente los valores en ayunas de cada medida posprandial $(10,11)$.

Se utilizó el test de Shapiro-Wilk para verificar la normalidad de las variables cuantitativas y, el de Levene, para examinar la igualdad de varianzas.

Las medidas de tendencia central se expresaron como promedio y desviación estándar (DE), o como medianas y rangos intercuartílicos (RIC), según el comportamiento de las variables.

Para las comparaciones entre grupos, se utilizaron las pruebas t de Student y la U de Mann-Whitney, y para las comparaciones previas y posteriores, la prueba t de Student en muestras relacionadas y la prueba de los rangos con signo de Wilcoxon para las variables paramétricas o no paramétricas.

Las correlaciones se establecieron calculando los coeficientes de correlación de Pearson o de
Spearman (ro). Para los cálculos, se usó el paquete estadístico SPSS ${ }^{\mathrm{TM}}$ (versión 21.0, SPSS Inc., Chicago, IL).

\section{Consideraciones éticas}

El proyecto fue sometido a evaluación y aprobación por parte de los comités de bioética de las Universidades de San Buenaventura de Cartagena e ICESI de Cali. Todos los participantes firmaron el consentimiento informado antes de comenzar el estudio. Esta investigación forma parte de un macroproyecto cuyo objetivo es determinar el impacto del agregado de aceite de Sacha Inchi a una comida rica en ácidos grasos saturados, registrado en ClinicalTrials.gov con el número NCT02886169.

\section{Resultados}

La muestra quedó conformada por 42 hombres, 21 con obesidad abdominal, cuyas edades fluctuaban entre 38,7 $\pm 10,1$ años (grupo sin obesidad) y 41,4 \pm 7,6 años (grupo con obesidad), sin diferencia significativa $\left(p=0,339 ; I C_{95 \%}:-8,2\right.$ a 2,9$)$. En ese mismo orden, los índices de masa corporal (IMC) fueron de $23,0 \pm 2,2 \mathrm{~kg} / \mathrm{m}^{2}$ y $30,1 \pm 4,1 \mathrm{~kg} /$ $\mathrm{m}^{2}\left(\mathrm{p}=0,001, \mathrm{IC}_{95 \%}:-9,1\right.$ a $\left.-5,0\right)$ y, los perímetros de cintura, de $85,0 \pm 6,3 \mathrm{~cm}$ y $103,2 \pm 8,9 \mathrm{~cm}$ $\left(p=0,001 ; I_{95 \%}:-23,0\right.$ a $\left.-13,4\right)$.

Los valores de glucosa, de insulina y el índice HOMA-IR en ayunas, así como el impacto de la ingestión, se presentan en el cuadro 1. La glucemia en ayunas no superó los límites normales en ninguno de los participantes, aunque mostró una ligera tendencia a valores mayores en el grupo con obesidad, diferencia que alcanzó significación estadística a la hora. La insulinemia registró valores mayores en los participantes con obesidad

Cuadro 1. Niveles de glucosa e insulina en ayunas y respuestas posprandiales tras la ingestión de una comida rica en grasas saturadas en el grupo sin obesidad abdominal $(n=21)$ y el grupo con obesidad abdominal $(n=21)$

\begin{tabular}{|c|c|c|c|c|c|}
\hline Parámetro & & $\begin{array}{c}\text { NO } \\
\text { Promedio (DE) }\end{array}$ & $\stackrel{0}{\text { Promedio (DE) }}$ & $\begin{array}{c}I_{C_{95 \%}} \text { dif } \\
\text { (NO Vs. O) }\end{array}$ & $\begin{array}{c}p \\
\text { (NO Vs. O) }\end{array}$ \\
\hline \multirow[t]{4}{*}{ Glucosa (mg/dl) } & Ayunas & $85,1 \quad(8,8)$ & $89,7 \quad(6,7)$ & $-9,5$ a $\quad 0,3$ & 0,065 \\
\hline & 1 hora & $91,9(16,9)$ & $106,0^{*}(12,5)$ & $-23,4$ a $\quad-4,8$ & 0,004 \\
\hline & 4 horas & $84,4(11,3)$ & $82,7^{*} \quad(9,6)$ & $-6,8$ a $\quad 6,3$ & 0,936 \\
\hline & $A \cup C$ & $350,0(43,5)$ & $380,8 \quad(33,0)$ & $-54,9$ a $-6,8$ & 0,013 \\
\hline \multirow[t]{4}{*}{ Insulina ( $\mu \mathrm{UI} / \mathrm{ml})$} & Ayunas & $10,3 \quad(7,8)$ & $17,6 \quad(7,9)$ & $-12,1$ a $-2,3$ & 0,005 \\
\hline & 1 hora & $28,9^{*}(16,4)$ & $71,6^{*}(40,6)$ & $-62,2$ a $-23,5$ & 0,001 \\
\hline & 4 horas & $9,2 \quad(6,7)$ & $13,0^{*} \quad(7,7)$ & $-8,3$ a $\quad 0,7$ & 0,096 \\
\hline & $A \cup C$ & $76,8(40,6)$ & $171,5(87,5)$ & $-137,3$ a $-52,3$ & 0,001 \\
\hline Índice HOMA-IR & Ayunas & $2,2(1,7)$ & $3,9 \quad(1,8)$ & $-2,8$ a $\quad-0,6$ & 0,004 \\
\hline
\end{tabular}

NO: grupos sin obesidad abdominal; O: grupo con obesidad abdominal; AUC: área bajo la curva; DE: desviación estándar

${ }^{*} p<0,005$ comparado con el valor en ayunas 
en todos los tiempos evaluados. El incremento del área bajo la curva de insulina fue menor en el grupo sin obesidad (iAUC=32,6 $\mu \mathrm{Ul} / \mathrm{ml} ; \mathrm{RIC}=20,2-$ $43,1)$ que en el grupo con obesidad (iAUC $=82,7$ $\mu \mathrm{Ul} / \mathrm{ml} ; \mathrm{RIC}=40,2-133,9 ; p=0,001$ ), pero no se halló diferencia para la glucosa, en tanto que las áreas totales bajo la curva fueron mayores para ambos parámetros en el grupo con obesidad (cuadro 1).

En lo concerniente a las variables lipídicas de triacilglicéridos, colesterol total y HDL-C, solo se registró diferencia entre los grupos en la concentración basal de los primeros, y fue mayor en el grupo con obesidad. El colesterol total y los triacilglicéridos aumentaron luego de la intervención, y se mantuvieron durante todo el tiempo que duró el ensayo, tal como se aprecia en el cuadro 2.

Las concentraciones de triacilglicéridos en ayunas se correlacionaron con los valores a las cuatro horas ( $r=0,854 ; p<0,001)$, con el AUC de los triacilglicéridos $(r=0,936 ; p<0,001)$, así como con el iAUC $(r=0,474 ; p<0,001)$.

Al comparar los grupos clasificados según la presencia de obesidad abdominal, el iAUC de los triacilglicéridos fue similar; sin embargo, cuando los participantes se reclasificaron según los niveles de triacilglicéridos, el grupo con niveles en ayunas superiores a $150,0 \mathrm{mg} / \mathrm{dl} \quad(\mathrm{n}=16 ; 38 \%)$ registró un iAUC $(179,3 \mathrm{mg} / \mathrm{dl} ; \mathrm{RIC}=123,6-283,9)$ significativamente mayor que en los restantes 26 sujetos ( $n=26 ; 62 \%$ ), cuyos niveles estuvieron por debajo de ese valor (iAUC=113,3 mg/dl; RIC=41,1197,$6 ; p=0,041$ ).

En el cuadro 3 se presentan los valores basales y posprandiales de los marcadores inflamatorios para ambos grupos. La proteína $\mathrm{C}$ reactiva en ayunas y a las cuatro horas fue mayor en el grupo con obesidad. Tras la ingestión, la IL-6 disminuyó en el grupo sin obesidad y los lipopolisacáridos aumentaron en ambos grupos. Los niveles de triacilglicéridos y de lipopolisacáridos a las cuatro horas, registraron una correlación positiva significativa solamente en el grupo con obesidad $(r=0,635 ; p=0,002)$.

\section{Discusión}

En los dos grupos se registraron concentraciones similares de glucemia basal (inferiores a $100 \mathrm{mg} /$ dl); sin embargo, la cuantificación de la insulina y el cálculo del índice HOMA-IR en ayunas evidenciaron el estado de resistencia a la insulina en el grupo con obesidad. Además, el reto alimentario permitió mostrar el aumento de las alteraciones fisiopatológicas en la homeostasis de este grupo, lo que otorga al momento posprandial una relevancia especial en la detección oportuna y temprana de este tipo de alteraciones (12), y permite ilustrar cuán inadecuado resulta descartar alteraciones del metabolismo de los glúcidos con base en determinaciones aisladas de glucemia en ayunas.

Cuadro 2. Niveles lipídicos en ayunas y respuestas posprandiales tras la ingestión de una comida rica en grasas saturadas en el grupo sin obesidad abdominal $(n=21)$ y el grupo con obesidad abdominal $(n=21)$

\begin{tabular}{|c|c|c|c|c|c|c|c|c|c|c|}
\hline \multirow[t]{2}{*}{ Parámetro } & \multirow[t]{2}{*}{ Tiempo } & \multicolumn{3}{|c|}{$\begin{array}{c}\text { NO } \\
\text { (Rangos intercuartílicos) }\end{array}$} & $\mathbf{p}$ & \multicolumn{3}{|c|}{$\begin{array}{c}0 \\
\text { (Rangos intercuartílicos) }\end{array}$} & \multirow{2}{*}{$\begin{array}{c}\mathbf{p} \\
\begin{array}{c}\text { Tiempo } V s . \\
\text { ayunas }\end{array}\end{array}$} & \multirow{2}{*}{$\begin{array}{c}\text { p } \\
\text { O Vs. NO }\end{array}$} \\
\hline & & 25 & 50 & 75 & $\begin{array}{l}\text { Tiempo } V s . \\
\text { ayunas }\end{array}$ & 25 & 50 & 75 & & \\
\hline \multirow[t]{5}{*}{ TAG (mg/dl) } & Ayunas & 70,0 & 99,0 & 152,5 & NA & 106,5 & 154,0 & 180,5 & NA & 0,044 \\
\hline & 1 hora & 90,5 & 121,0 & 189,5 & 0,001 & 130,5 & 178,0 & 215,5 & 0,001 & 0,099 \\
\hline & 4 horas & 87,0 & 164,0 & 231,0 & 0,001 & 143,0 & 210,0 & 301,5 & 0,001 & 0,080 \\
\hline & iAUC & 81,3 & 134,5 & 201,0 & NA & 46,8 & 147,5 & 278,8 & NA & 0,546 \\
\hline & $A \cup C$ & 360,0 & 570,0 & 770,8 & NA & 531,2 & 744,0 & 917,3 & NA & 0,046 \\
\hline \multirow[t]{5}{*}{ CT (mg/dl) } & Ayunas & 141,8 & 156,9 & 184,6 & NA & 153,5 & 177,1 & 193,3 & NA & 0,222 \\
\hline & 1 hora & 139,4 & 161,0 & 185,9 & 0,237 & 158,7 & 176,3 & 202,5 & 0,033 & 0,222 \\
\hline & 4 horas & 151,4 & 167,5 & 189,2 & 0,018 & 151,8 & 179,5 & 201,0 & 0,017 & 0,365 \\
\hline & iAUC & $-2,3$ & 13,5 & 25,3 & NA & 0,9 & 14,0 & 35,4 & NA & 0,589 \\
\hline & AUC & 572,6 & 673,6 & 726,1 & NA & 623,1 & 718,9 & 798,7 & NA & 0,232 \\
\hline \multirow[t]{5}{*}{ HDL-C (mg/dl) } & Ayunas & 41,2 & 47,9 & 56,6 & NA & 41,8 & 47,8 & 54,4 & NA & 0,880 \\
\hline & 1 hora & 42,6 & 48,8 & 55,5 & 0,732 & 44,5 & 48,3 & 55,1 & 0,006 & 0,782 \\
\hline & 4 horas & 44,3 & 47,2 & 57,9 & 0,033 & 43,1 & 47,7 & 53,9 & 0,348 & 0,606 \\
\hline & iAUC & $-2,2$ & 5,4 & 13,3 & NA & $-1,2$ & 3,2 & 11,5 & NA & 0,606 \\
\hline & $A \cup C$ & 174,7 & 190,5 & 218,1 & NA & 175,9 & 189,3 & 220,9 & NA & 0,890 \\
\hline
\end{tabular}

NO: grupos sin obesidad abdominal; O: grupo con obesidad abdominal; TAG: triacilglicéridos; CT: colesterol total; HDL-C: colesterol de lipoproteínas de alta densidad; iAUC: incremento del área bajo la curva; AUC: área bajo la curva; NA: no aplica 
Cuadro 3. Concentraciones de los marcadores inflamatorios en ayunas y posprandiales en el grupo sin obesidad abdominal ( $n=21$ ) y el grupo con obesidad abdominal $(n=21)$

\begin{tabular}{|c|c|c|c|c|c|c|c|c|c|c|}
\hline \multirow[t]{2}{*}{ Parámetro } & \multirow[t]{2}{*}{ Tiempo } & \multicolumn{3}{|c|}{$\begin{array}{c}\text { NO } \\
\text { (Rangos intercuartílicos) }\end{array}$} & $\mathbf{p}$ & \multicolumn{3}{|c|}{$\begin{array}{c}0 \\
\text { (Rangos intercuartílicos) }\end{array}$} & $\mathbf{p}$ & $\mathbf{p}$ \\
\hline & & 25 & 50 & 75 & $\begin{array}{l}\text { Tiempo } V s . \\
\text { ayunas }\end{array}$ & 25 & 50 & 75 & $\begin{array}{l}\text { Tiempo } V s . \\
\text { ayunas }\end{array}$ & O Vs. NO \\
\hline \multirow[t]{2}{*}{ IL-6 (pg/ml) } & Ayunas & 0,61 & 1,41 & 2,03 & NA & 1,07 & 1,44 & 2,41 & NA & 0,489 \\
\hline & 4 horas & 0,48 & $0,87^{*}$ & 2,10 & 0,042 & 1,10 & 1,39 & 2,21 & 0,543 & 0,182 \\
\hline \multirow[t]{2}{*}{ PCR-us (mg/l) } & Ayunas & 0,50 & 0,95 & 1,70 & NA & 1,21 & 2,11 & 2,90 & NA & 0,009 \\
\hline & 4 horas & 0,46 & 1,04 & 1,70 & 0,173 & 1,62 & 2,02 & 2,76 & 0,114 & 0,002 \\
\hline \multirow[t]{3}{*}{ LPS (EU/ml) } & Ayunas & 0,27 & 0,49 & 1,09 & NA & 0,26 & 0,38 & 0,92 & NA & 0,497 \\
\hline & 1 hora & 0,19 & 0,60 & 1,04 & 0,986 & 0,38 & 0,70 & 1,05 & 0,122 & 0,615 \\
\hline & 4 horas & 0,72 & $1,33^{* *}$ & 1,72 & 0,005 & 0,67 & 1,06 & $1,70^{* *}$ & 0,004 & 0,513 \\
\hline
\end{tabular}

NO: grupos sin obesidad abdominal; O: grupo con obesidad abdominal; IL-6: interleucina 6; PCRus: proteína C reactiva ultrasensible; LPS: lipopolisacáridos; NA: no aplica; ${ }^{*} p<0,05 ;{ }^{* *} p<0,01$ comparado con el valor en ayunas

Es interesante señalar que el mayor incremento de las concentraciones de glucosa e insulina en el grupo con obesidad en el momento posprandial cayó a las cuatro horas a valores menores que los del ayuno, situación que no se observó en el grupo sin obesidad y que coincide con lo hallado por Rubin, et al., en el 2010 (13). Este comportamiento alerta sobre fallas en la regulación de la insulina y sobre el exceso de secreción posterior al estímulo, eventos que contribuyen al agotamiento del páncreas y favorecen los efectos derivados de la hiperinsulinemia, reflejando la pérdida de la capacidad de restaurar la homeostasis, que en la actualidad se considera incluida en el concepto de salud como la habilidad para adaptarse y manejar de manera autónoma los retos de la ingestión de alimentos (14).

El impacto específico derivado de la intervención, medido como iAUC, fue similar entre los grupos para la glucosa pero no para la insulina, lo cual demuestra el esfuerzo pancreático del grupo con obesidad para controlar la glucemia, en tanto que el aumento global (AUC) fue mucho mayor en presencia de obesidad para una y otra, lo cual pone en evidencia la sobrecarga bioquímica que acompaña la alteración metabólica en este grupo.

A diferencia de lo hallado para la glucemia, en el cuadro 2 se evidencia que las concentraciones de triacilglicéridos en ayunas fueron mayores en el grupo con obesidad y que los valores posprandiales de triacilglicéridos y colesterol total se mantuvieron por encima de los del ayuno en todos los tiempos de evaluación en ambos grupos, lo cual se asemeja a lo reportado en otros estudios (15). Es importante destacar que, si se asumen frecuencias alimentarias de cuatro horas en promedio y un consumo de alimentos ricos en grasas saturadas en un día normal, un individuo no lograría regresar a sus valores lipídicos en ayunas durante la mayor parte del día. Esto evidencia que la medición de las concentraciones no basales, especialmente de triacilglicéridos y colesterol total, permite verificar la respuesta metabólica a la ingestión y el estado más habitual en un día normal, aun cuando pueda resultar más complicada desde un punto de vista operativo.

Además, aunque el papel de los triacilglicéridos como factor de riesgo cardiovascular ha sido objeto de controversia (16), en estudios clínicos recientes se ha evidenciado que, especialmente, los niveles posprandiales se relacionan con la enfermedad coronaria (17).

En este estudio, se registraron diferencias en los triacilglicéridos en ayunas entre los grupos clasificados según la obesidad, pero similares respuestas posprandiales, a diferencia de otras investigaciones que demostraron aumentos mayores en los obesos que en quienes no lo eran, aunque la medición se hizo en los grupos cuyos triacilglicéridos en ayunas estaban en los niveles normales (18).

Por ello, en el presente estudio se decidió reclasificar los grupos según la presencia de niveles alterados de triacilglicéridos en ayunas ( $\geq 150,0 \mathrm{mg} / \mathrm{dl})$, lo cual permitió demostrar que, en el grupo cuyos niveles eran elevados, los incrementos posteriores a la ingestión eran mayores. Estos hallazgos, sumados a la significativa correlación entre ambos valores, avalan lo expresado por Almeda, et al., en el sentido de que la concentración basal de los triacilglicéridos representa uno de los principales factores determinantes de la lipemia posprandial (19). 
En estudios previos en adultos con sobrepeso, la ingestión de un desayuno de 1.416 calorías (52\% de las cuales provenía de grasas) resultó en incrementos del colesterol total y de los triacilglicéridos, y en un aumento de los marcadores inflamatorios y de los lipopolisacáridos (20). Según la información disponible, estos lipopolisacáridos se transportarían ligados a lipoproteínas ricas en triacilglicéridos y participarían en procesos de inflamación y de expresión de citocinas y moléculas de adhesión, contribuyendo así al desarrollo de enfermedades cardiovasculares (21-24). En el presente estudio se encontró que los valores basales de los lipopolisacáridos y de la IL-6 fueron similares en ambos grupos, pero su comportamiento posprandial difirió, como puede observarse en el cuadro 3 . En cuanto a la proteína $\mathrm{C}$ reactiva, la concentración basal fue mayor en el grupo con obesidad, lo cual evidenció el proceso crónico, sin variación posterior a la ingestión, lo que parece indicar que no responde en los tiempos usuales de una intervención aguda, como se ha hallado en otros estudios $(3,25)$.

En cuanto a la IL-6, esta había presentado resultados contradictorios en estudios anteriores, lo que llevó a postular que su aumento podía deberse a variaciones fisiológicas (26) o a efectos derivados de la inflamación local por las punciones repetidas o el uso de catéter $(27,28)$. Las precauciones en la toma de la muestra que ya se explicaron garantizan la validez de los resultados de este estudio y evidencian que la caída en los niveles de la IL-6 en el grupo sin obesidad reflejaría un menor impacto inflamatorio en este grupo, lo cual coincide con lo hallado por otros autores (29).

Los lipopolisacáridos aumentaron en ambos grupos en la etapa posprandial, lo cual se ha descrito en diversos estudios como relacionado con el incremento de los triacilglicéridos en el grupo con obesidad, y era de esperarse dadas las evidencias de que los lipopolisacáridos entran en circulación mediados por lipoproteínas ricas en triacilglicéridos (30). El aumento de los lipopolisacáridos en la etapa posprandial en el grupo sin obesidad, discrepa de lo hallado en otros estudios (23), lo que podría deberse a la mayor edad de nuestros participantes, circunstancia que ha demostrado afectar las reacciones posprandiales (31).

Por otra parte, la ausencia de correlación entre los niveles de triacilglicéridos y lipopolisacáridos en este mismo grupo, podría deberse a diferencias derivadas del tipo de alimentación y a los niveles previos de translocación de los lipopolisacáridos, así como al desarrollo de la memoria adaptativa inmunitaria o a la presencia de proteínas de transporte que podrían enmascarar el aumento en los valores basales o posprandiales $(32,33)$, o reflejarse en habilidades diferentes para el manejo de los incrementos de endotoxinas (34), aspectos que deberán abordarse en el futuro.

A diferencia de otros estudios que ensayan cargas lipídicas en forma de batidos y con seguimiento a las ocho horas, en este ensayo se incluyó un alimento similar en contenido y consistencia al consumido habitualmente por los participantes y se hizo seguimiento a las cuatro horas, considerado como el tiempo promedio entre comidas, lo cual otorga a nuestro estudio la fortaleza de asegurar tiempos de vaciado gástrico y digestión similares a una ingestión común, que podrían alterarse con el consumo de cargas grasas exclusivamente líquidas. La aleatorización del muestreo, las indicaciones para la semana previa al estudio y el riguroso cumplimiento de los criterios de inclusión y exclusión, permitieron el control de variables derivadas de los estilos de vida.

Se requieren, sin embargo, estudios posteriores que incluyan mayor número de variables, especialmente del estado inflamatorio, así como intervenciones con diferentes ácidos grasos o seguimientos a largo plazo, para evaluar la ingestión crónica.

En suma, los resultados obtenidos muestran que la ingestión de una comida rica en ácidos grasos saturados modificó las variables relacionadas con el metabolismo de los glúcidos en ambos grupos, con mayor impacto en el grupo con obesidad al incrementar el estado de resistencia basal a la insulina. En cuanto a los lípidos, los dos grupos tuvieron una respuesta similar del colesterol, a diferencia de los triacilglicéridos, parámetro que aumentó mucho más en el grupo con obesidad, especialmente cuando se registraba mayor concentración en ayunas. El grupo sin obesidad registró menores niveles de inflamación basal y posprandial, según la estimación de las concentraciones de proteína $C$ reactiva en ayunas y la disminución de IL-6 después de la carga, respectivamente.

Cabe destacar que, aunque requiere de una mayor inversión de recursos y una mejor estandarización para fortalecer su valor clínico, la evaluación del estado posprandial refleja el estado más habitual de un día normal y hace posible evidenciar la respuesta metabólica a la alimentación, lo que 
contribuye a detectar los trastornos metabólicos tempranos relacionados con la presencia de obesidad abdominal en personas aparentemente sanas.

\section{Conflicto de intereses}

Los autores declaran no tener conflictos de intereses.

\section{Financiación}

Este proyecto se financió con recursos provenientes de las convocatorias internas de la Universidad ICESI (Cali) y de la Universidad de San Buenaventura (Cartagena).

\section{Referencias}

1. Wopereis S, Wolvers D, van Erk M, Gribnau M, Kremer B, van Dorsten FA, et al. Assessment of inflammatory resilience in healthy subjects using dietary lipid and glucose challenges. BMC Med Genomics. 2013;6:1-16. https://doi. org/10.1186/1755-8794-6-44

2. Laugerette F, Vors C, Peretti N, Michalski MC. Complex links between dietary lipids, endogenous endotoxins and metabolic inflammation. Biochimie. 2011;93:39-45. https:// doi.org/10.1016/j.biochi.2010.04.016

3. Schwander F, Kopf KA, Buri C, Portmann R, Egge $\mathrm{L}$, Chollet $\mathrm{M}$, et al. A dose-response strategy reveals differences between normal-weight and obese men in their metabolic and inflammatory responses to a high-fat meal. Fat Meal J Nutr. 2014;144:1517-23. https://doi.org/10.3945/ jn.114.193565

4. Al-Disi DA, Al-Daghri NM, Khan N, Alfadda AA, Sallam RM, Alsaif M, et al. Postprandial effect of a high-fat meal on endotoxemia in Arab women with and without insulinresistance-related diseases. Nutrients. 2015;7:6375-89. https://doi.org/10.3390/nu7085290

5. Maffeis C, Pinelli L, Surano MG, Fornari E, Cordioli $\mathrm{S}$, Gasperotti $\mathrm{S}$, et al. Pro-atherogenic postprandial profile: Meal-induced changes of lipoprotein sub-fractions and inflammation markers in obese boys. Nutr Metab Cardiovasc Dis.2012;22:959-65. https://doi.org/10.1016/j. numecd.2010.12.009

6. Instituto Colombiano de Bienestar Familiar. Encuesta Nacional de la Situación Nutricional en Colombia, 2010. Resumen ejecutivo ENSIN 2010. Fecha de consulta: 12 de enero de 2017. Disponible en: http://www.icbf.gov.co/portal/ page/portal/Descargas1/Resumenfi.pdf

7. Ministerio de Salud y Protección Social. Análisis de Situación de Salud, 2015. Fecha de consulta: 12 de enero de 2017. Disponible en: https://www.minsalud.gov.co/sites/ rid/Lists/BibliotecaDigital/RIDE/VS/ED/PSP/asis-2015.pdf

8. Departamento Administrativo Distrital de Salud, Cartagena. Perfil epidemiológico del Distrito de Cartagena año 2014. Fecha de consulta: 3 de enero de 2017. Disponible en: http://www.dadiscartagena.gov.co/images/ docs/saludpublica/perfil_epidemiologico_2014.pdf

9. Sáenz $\mathbf{S}$, González $\mathbf{F}$, Díaz $\mathbf{S}$. Hábitos y trastornos alimenticios asociados a factores socio-demográficos, físicos y conductuales en universitarios de Cartagena, Colombia. Rev Clin Med Fam. 2011;4:193-204. https://doi.org/10.4321/ S1699-695X2011000300003
10. Guerci B, Paul JL, Hadjadj S, Durlach V, Vergés B, Attia $\mathbf{N}$, et al. Analysis of the postprandial lipid metabolism: Use of a 3-point test. Diabetes Metab. 2001;27:449-57. https:// doi.org/DM-09-2001-27-4-1262-3636-101019-ART4

11. Carstensen $\mathbf{M}$, Thomsen $\mathbf{C}$, Hermans $\mathbf{K}$. Incremental area under response curve more accurately describes the triglyceride response to an oral fat load in both healthy and type 2 diabetic subjects. Metabolism. 2003;52:1034-7. https://doi.org/10.1016/S0026-0495(03)00155-0

12. Patarrão RS, Lautt WW, Afonso RA, Ribeiro RT, Fernandes AB, Boavida JM, et al. Postprandial but not fasting insulin resistance is an early identifier of dysmetabolism in overweight subjects. Can J Physiol Pharmacol. 2012;90:923-31. https://doi.org/10.1139/y2012-086

13. Rubin D, Helwig U, Nothnagel M, Folsch UR, Schreiber S, Schrezenmeir J. Association of postprandial and fasting triglycerides with traits of the metabolic syndrome in the Metabolic Intervention Cohort Kiel. Eur J Endocrinol. 2010;162:719-27. https://doi.org/10.1530/EJE-09-0446

14. Kardinaal AF, van Erk MJ, Dutman AE, Stroeve JH, van de Steeg E, Bijlsma S, et al. Quantifying phenotypic flexibility as the response to a high-fat challenge test in different states of metabolic health. FASEB J. 2015;29:460013. https://doi.org/10.1096/fj.14-269852

15. Silliman K, Mahoney J, Michels V, Miles T. No apparent impact of meal glycemic index on postprandial lipid response in lean and abdominally obese women. Open Nutr J. 2012;6: 97-103. https://doi.org/10.2174/1874288201206010097

16. Singh AK, Singh R. Triglyceride and cardiovascular risk: A critical appraisal. Indian J Endocrinol Metab. 2016;20:41828. https://doi.org/10.4103/2230-8210.183460

17. Manochehri M, Moghadam AJ. Studying the relation of postprandial triglyceride with coronary artery disease (CAD). Med Arch. 2016;70:261-4. https://doi.org/10.5455/ medarh.2016.70.261-264

18. Nogaroto V, Rodrigues MR, Vicari MR, De Almeida MC, Milléo FQ, Dos Santos FA, et al. High postprandial triglycerides serum levels: Is obesity a good predictor? An Acad Bras Ciênc. 2015;87:437-45. https://doi.org/10.1590/0 0013765201520130380

19. Almeda P, Cuevas D, Mehta R, Muñoz L, Cruz I, Pérez $\mathbf{O}$, et al. Factors associated with postprandial lipemia and apolipoprotein A-V levels in individuals with familial combined hiperlipidemia. BMC Endocr Disord. 2014;14:90. https://doi.org/10.1186/1472-6823-14-90

20. Miglio C, Villan DV, Toti E, Peluso I, Raguzzini A, Cesqui $E$, et al. Antioxidant and inflammatory response following high-fat meal consumption in overweight subjects. Eur $\mathrm{J}$ Nutr. 2013;52:1107-14. https://doi.org/10.1007/s00394-0120420-7

21. Den Hartigh LJ, Altman R, Norman JE, Rutledge JC. Postprandial VLDL lipolysis products increase monocyte adhesion and lipid droplet formation via activation of ERK2 and NFkB. Am J Physiol Heart Circ Physiol. 2014;306: H109-20. https://doi.org/10.1152/ajpheart.00137.2013

22. Ghoshal S, Witta J, Zhong J, de Villiers W, Eckhardt E. Chylomicrons promote intestinal absorption of lipopolysaccharides. J Lipid Res. 2009;50:90-7. https://doi. org/10.1194/jlr.M800156-JLR200 
23. Vors C, Pineau G, Drai J, Meugnier E, Pesenti S, Laville M, et al. Postprandial endotoxemia linked with chylomicrons and LPS handling in obese vs lean men: A lipid-dose effect trial. J Clin Endocrinol Metab. 2015;100:3427-35. https:// doi.org/10.1210/JC.2015-2518

24. Wang YI, Schulze J, Raymond N, Tomita T, Tam K, Simon SI, et al. Endothelial inflammation correlates with subject triglycerides and waist size after a high-fat meal. Am J Physiol Heart Circ Physiol. 2011;30:H784-91. https://doi. org/10.1152/ajpheart.01036

25. Wagmacker D, Petto J, Leichsenring F, Nery AC, Teixeira AM. C-reactive protein in the initial phase of postprandial lipemia in subjects with central obesity. Int J Cardiovasc Sci. 2015;28:9-15. https://doi.org/10.5935/2359-4802.20150003

26. Gregersen S, Samocha D, Heilbronn LK, Campbell LV. Inflammatory and oxidative stress responses to highcarbohydrate and high-fat meals in healthy humans. J Nutr Metab. 2012. https://doi.org/10.1155/2012/238056

27. Herieka M, Erridge C. High-fat meal induced postprandial inflammation. Mol Nutr Food Res. 2014;58:136-46. https:// doi.org/10.1002/mnfr.201300104

28. Haack M, Kraus T, Schuld A, Dalal M, Koethe D, Pollmacher T. Diurnal variations of interleukin-6 plasma levels are confounded by blood drawing procedures. Psychoneuroendocrinology. 2002;27:921-31. https://doi.org/10.1016/ S0306-4530(02)00006-9
29. Tholstrup T, Teng K, Raff M. Dietary cocoa butter or refined olive oil does not alter postprandial hsCRP and IL-6 concentrations in healthy women. Lipids. 2011;46:365-70. https://doi.org/10.1007/s11745-011-3526-4

30. Ghanim H, Sia CL, Upadhyay M, Korzeniewski K, Viswanathan $\mathrm{P}$, Abuaysheh $\mathrm{S}$, et al. Orange juice neutralizes the proinflammatory effect of a high-fat, highcarbohydrate meal and prevents endotoxin increase and Toll-like receptor expression. Am J Clin Nutr. 2010;91:9409. https://doi.org/10.3945/ajcn.2009.28584

31. Zaman GF, Zaman F, Raul MG. Comparison of postprandial endotoxemia in male adolescent and male subjects above 50 years after a fat overload. Int J Curr Res Aca Rev. 2014;2:167-74.

32. Luck H, Tsai S, Chung J, Clemente X, Ghazarian M, Revelo XS, et al. Regulation of obesity-related insulin resistance with gut anti-inflammatory agents. Cell Metabolism. 2015; 21:527-42. https://doi.org/10.1016/j.cmet.2015.03.001

33. Laugerette F, Alligier M, Bastard JP, Drai J, Chanseaume E, Lambert S, et al. Overfeeding increases postprandial endotoxemia in men: Inflammatory outcome may depend on LPS transporters LBP and SCD14. Mol Nutr Food Res. 2014;58:1513-8. https://doi.org/10.1002/mnfr.201400044

34. Boutagy NE, McMillan RP, Frisard MI, Hulver MW. Metabolic endotoxemia with obesity: Is it real and is it relevant? Biochimie. 2016;124:11-20. https://doi.org/10. 1016/j.biochi.2015.06.020 